\title{
Laser Processing by Using Multi Level Free Form 3D Micro-fabricated DOE
}

\author{
Akihide HAMANO ${ }^{* 1}$, Atsushi YAMADA ${ }^{* 1}$, Takeshi TAKADA ${ }^{* 2}$, and Yoshiyuki USUKI ${ }^{* 1}$ \\ ${ }^{* 1}$ Material Research Laboratory, R\&D Division, Furukawa Co., Ltd., 1-25-13 Kannondai, Tsukuba, \\ Ibaraki 305-0856, Japan \\ a-hamano@furukawakk.co.jp \\ ${ }^{*_{2}}$ R\&D Planning Department, R\&D Division, Furukawa Co., Ltd., 2-2-3 Marunouchi, Chiyoda-ku, \\ Tokyo 100-8370, Japan
}

\begin{abstract}
A diffractive optical element (DOE) is a promising means of converting a single spot to multiple spots. The multi-level structures of the DOE exhibit a high performance. The DOE structure has the same shape as the multi-level free-form design. This DOE, made of $\mathrm{SiO}_{2}$, withstands a high power and peak laser and has a high transmittance in the visible and near-infrared region. Four split beams generated by the DOE are focused by a lens. The split spot size is similar to the spot size without DOE. The fluctuation of intensity of the four spots is obtained as $7.5 \%$. The distance between spots is similar to the design distance. Twenty-one spot simultaneous processing by a pico-second laser by using this DOE on a copper plate was demonstrated successfully for the first time. The processed pattern along the optical axis is in good agreement with the optical simulation.
\end{abstract}

DOI: $10.2961 /$ jlmn.2014.03.0011

Keywords: Diffractive optical element, Multi-level, 3D, Micro-structure, Laser processing, Picosecond

\section{Introduction}

Lasers are used in the modern world for a variety of processing techniques such as cutting and welding. The laser beam is typically focused on a spot by using a lens. However, simultaneous irradiation of multiple spots is desired as a laser processing technique [1-2]. A diffractive optical element (DOE) is a promising means of converting a single spot to multiple spots [1-3]. Femtosecond laser processing of multi-beams was reported using a DOE with four levels $[1,4]$. In the case of four and eight levels, the first-order diffraction efficiency is estimated as $81 \%$ and $95 \%$, respectively $[5,6]$. A high-efficiency DOE is desirable not only for first-order diffraction but also for high-order diffractive patterns for laser processing. It has been suggested that a DOE with more than four levels would offer higher performance [7].

A DOE with more than four levels is created using a free-form three-dimensional (3D) micro-scale structure. This DOE is fabricated in $\mathrm{SiO}_{2}$ substrate to withstand a high-power laser and have a high transmittance in the visible and near-infrared region. The generation of four spots is evaluated as having a high performance from incident single beams by using our multi-level free-form 3D micro-fabricated DOE. By using this DOE, multi-spot processing of a pico-second laser in a copper plate is demonstrated successfully.

The DOE with multi-level structure is introduced in section 2. The optical performance of multi-spot irradiation using the multi-level DOE described in section 3. The processing of a laser using DOE is demonstrated in section 4. Finally, section 5 summarizes our study.

\section{Multi-level free-form DOE}

The structural design of a multi-level DOE is shown in Fig. 1. The design of the DOE was calculated using Virtual $\mathrm{Lab}^{\mathrm{TM}}$ software (Light Trans $\mathrm{GmbH}$ ). The fabricated structure based on the structural design is shown in Fig. 2, and is measured by a 3D optical surface profiler (NewView 6200, Zygo Corporation). The fabricated structure is considered to have a similar shape to the structural design. The designed height $h_{\mathrm{pv}}$ (peak-tovalley of structure) along the $\mathrm{z}$ axis of the structure of DOE is given by [8]:

$$
h_{\mathrm{pv}}=\frac{\lambda}{n-1}
$$

where $\lambda$ is the wavelength, $n$ is the refractive index of $\mathrm{SiO}_{2}$, and $h_{\mathrm{pv}}$ depends on the wavelength $\lambda$.

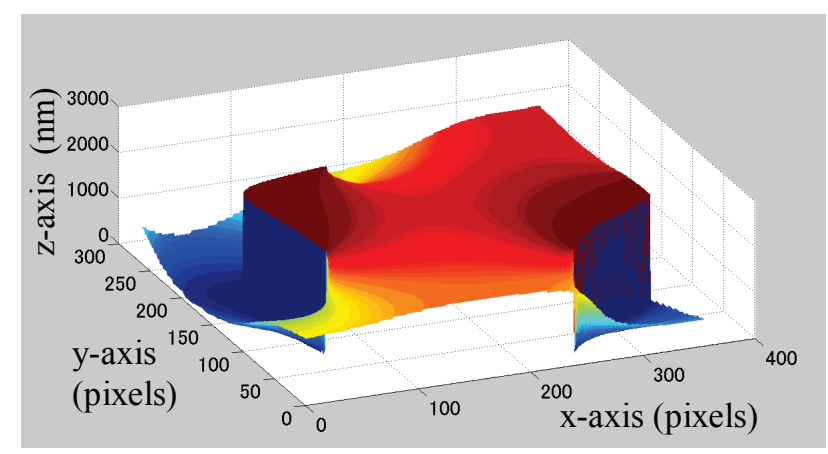

Fig. 1 Structural design of DOE. 


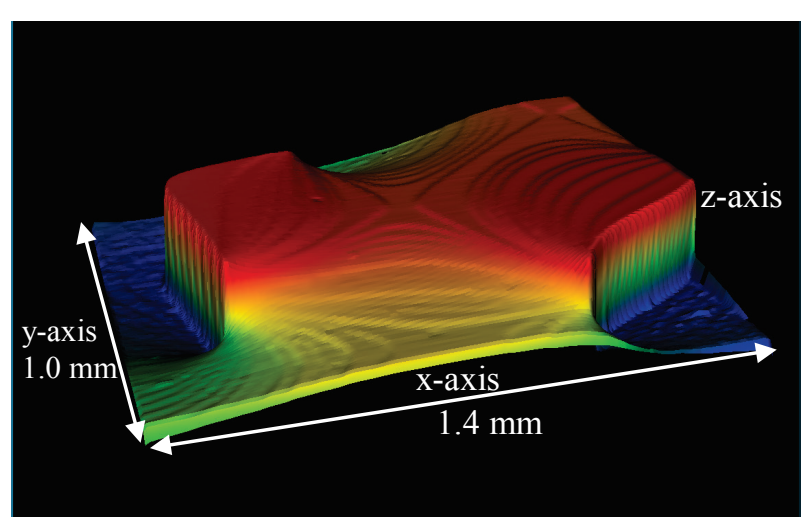

Fig. 2 Fabricated DOE structure.

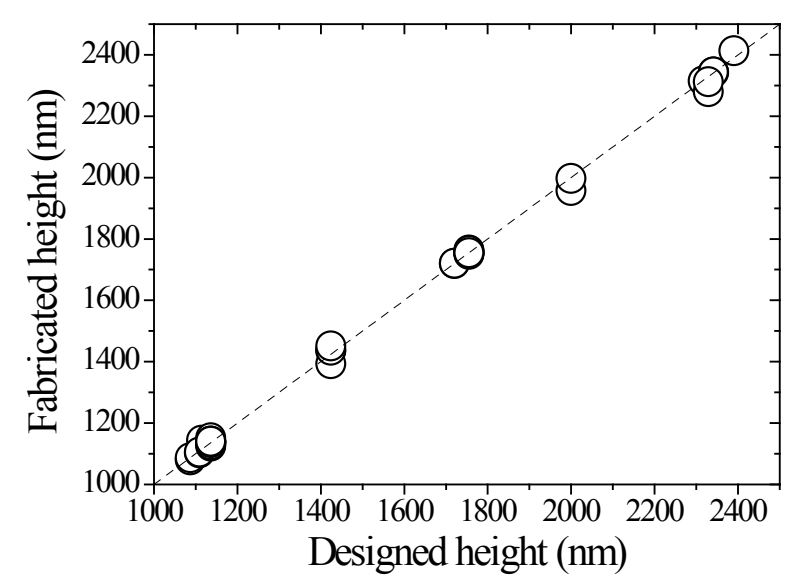

(a) Fabricated height with respect to designed height

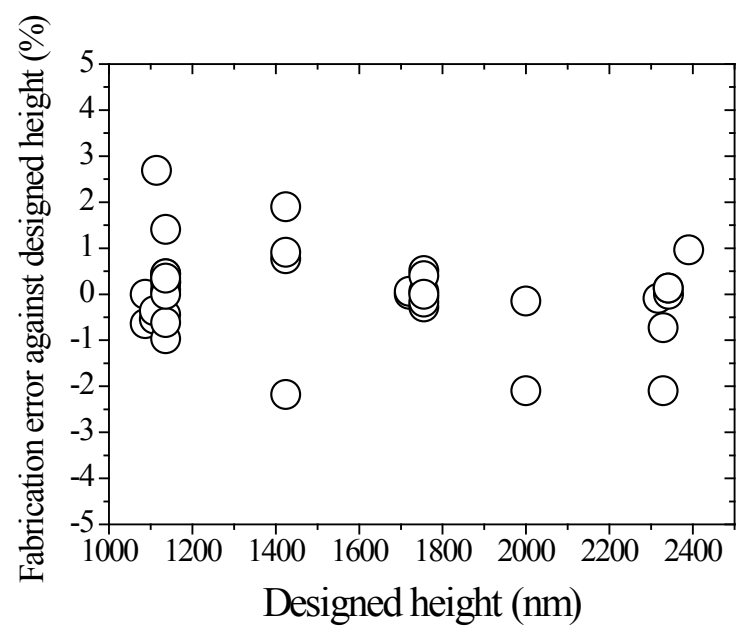

(b) Fabrication error against designed height

Fig. 3 Accuracy of fabricated height.

The fabricated height with respect to the designed height is indicated in Fig. 3(a). The fabricated height agrees well with the designed height.

Figure 3(b) shows the fabrication error plotted against the designed height $h_{\mathrm{pv}}$. We can fabricate the DOE within an error of less than $3 \%$. The standard deviation of the fabrication error is estimated as $0.9 \%$.

Using this DOE, we will demonstrate multi-spot irradiation with an arbitrary pattern.

\section{Optical performance of DOE}

A common optical setup with the DOE is shown in Fig. 5 using a laser and a lens. The laser beam is focused by the lens and split by the DOE.

The wavelength of the laser is $532 \mathrm{~nm}$, the diameter of the laser beam is $8 \mathrm{~mm}$, and the focal length is $1 \mathrm{~m}$. Four spots with a constant intensity are designed with a distance of $97 \mu \mathrm{m}$ among neighboring spots at the focal plane. The beam profile at the focal plane is obtained by a beam profiler (Laser Cam HR, Coherent, Inc.). Without the DOE setup, the beam profile of a focus spot is as shown in Fig. 6(a). A beam diameter of $\sim 90 \mu \mathrm{m}$ was observed. Next, a DOE is optically set up as shown in Fig. 5. A diffracted focus pattern of zigzag four spots is observed, with no noise (other the diffracted signal) except the four spots as shown in Fig. 6(b). The results of Figs. 7-9 were obtained from analyzing the data in Fig. 6.

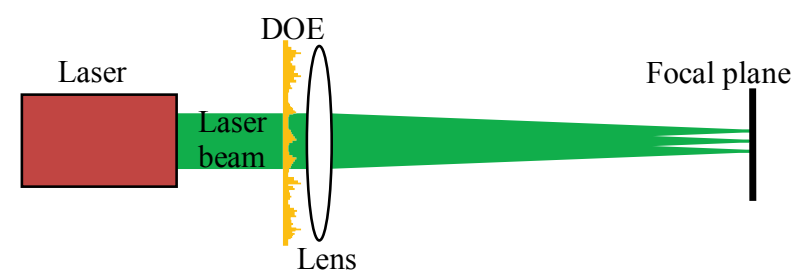

Fig. 5 Optical setup.

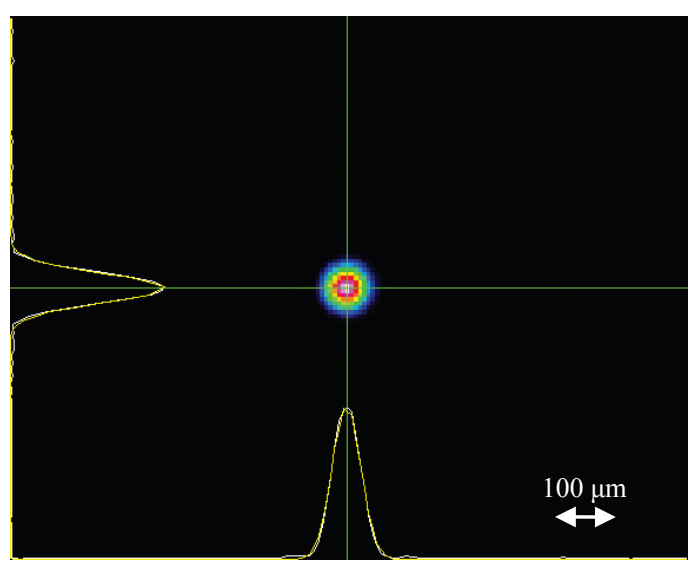

(a) Without DOE

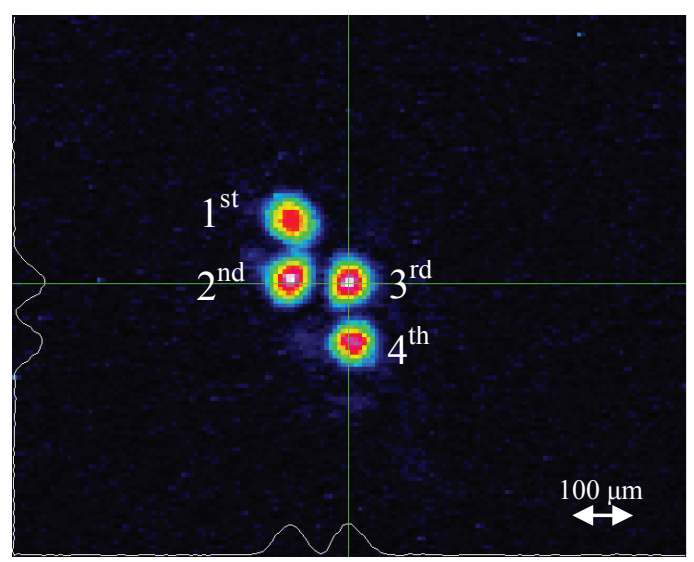

(b) With DOE

Fig. 6 Beam profile at focal plane. 


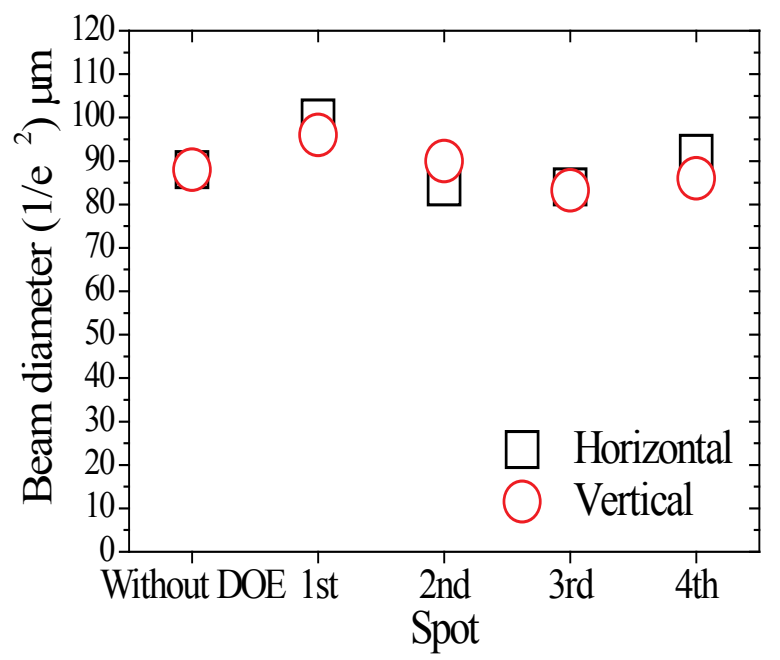

Fig. 7 Beam diameter at the focal plane.

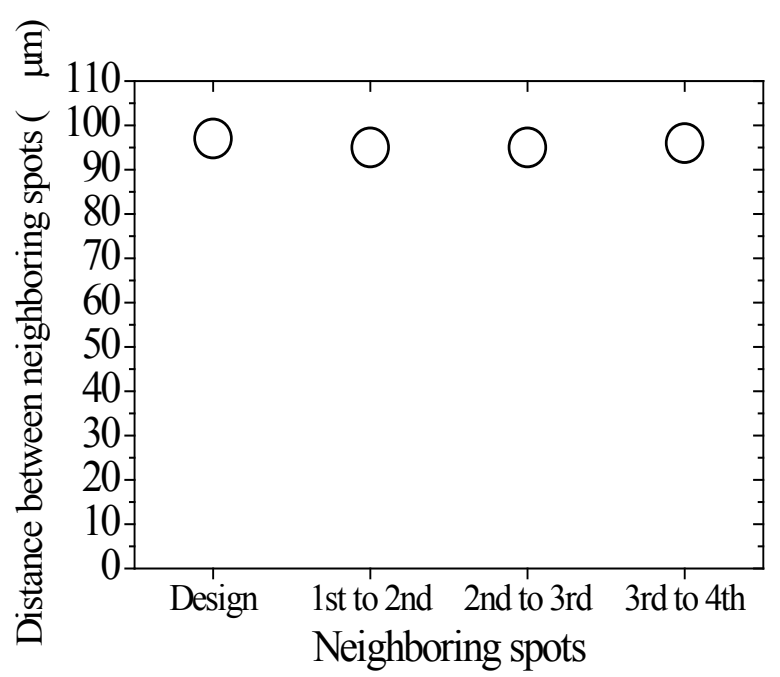

Fig. 8 Distance between neighboring spots.

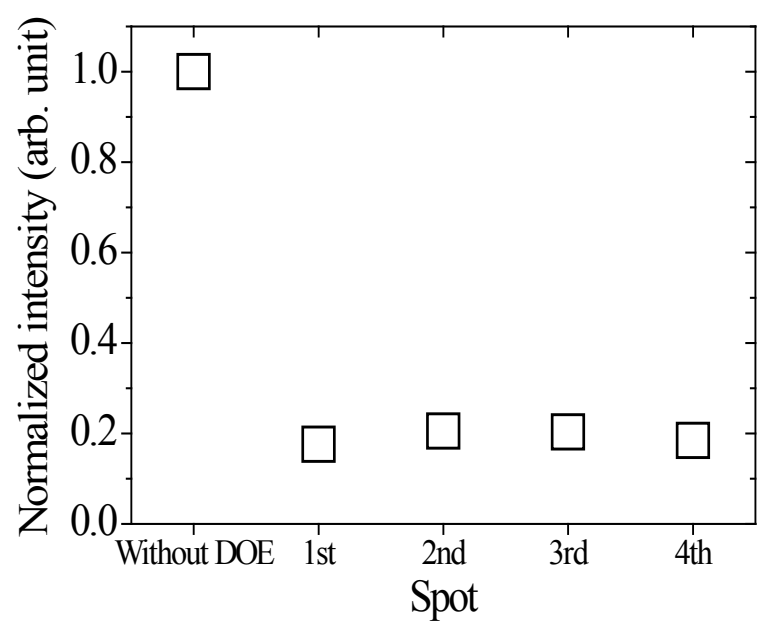

Fig. 9 Intensity of the focus spot.

The diameters of the four spots were obtained as $\sim 90 \mu \mathrm{m}$, as shown in Fig. 7. The diameter of the third spot is almost the same as that of the spot without DOE because the spatial position of the third spot is the same as that of the spot without DOE. The diameters of the other spots are slightly larger than that of the third spot. Distances between neighboring spots are similar to the results of the design shown in Fig. 8. The intensities of the focus spots are evaluated in Fig. 9. The intensities of the four spots are obtained as approximately $1 / 5$ of the intensity without DOE. Fluctuation (the ratio of standard deviation to the average) of the four spots is evaluated as $7.5 \%$.

We demonstrated high-efficiency multi-spot irradiation with arbitrary position. Our DOE offers high optical performance when using a high power and peak laser.

\section{Laser processing by using DOE}

The wavelength and pulse width of the laser are 1030 $\mathrm{nm}$ and $8 \mathrm{ps,}$ respectively, and the pulse repetition frequency is $2 \mathrm{kHz}$. The laser beam after transmission by the DOE is focused on the copper plate by a lens. The focal length of this lens is $100 \mathrm{~mm}$. The duration of laser processing is $1 \mathrm{~s}$. Twenty-one beams split by the DOE (different type than in section 3) are focused simultaneously on the copper plate. The experimental results are shown in Fig. 10(a) along with the optical axis ( $\mathrm{z}$ axis). A pattern of $\mathrm{z}=0 \mathrm{~mm}$ is obtained at the focused position. Twenty-one dots processed simultaneously by the pico-second laser were observed on the copper plate. $\mathrm{Up}$ to $+0.4 \mathrm{~mm}$, the dots are separated independently. At $+0.6 \mathrm{~mm}$ and $+0.8 \mathrm{~mm}$, the size of each spot is larger than that of the focused position ( $\mathrm{z}=0 \mathrm{~mm})$, due to the defocusing. Optical simulation patterns of multi-beams are shown in Fig. 10(b). The variation of processed pattern along the $\mathrm{z}$-axis in the experimental result is similar to the variation of pattern of the optical simulation. The $200-\mu \mathrm{m}$ processed pattern is the same size as the optical simulation. Thus, pico-second laser processing of multi-spots on a copper plate was successfully demonstrated using the multi-level free-form 3D micro-fabricated DOE.

\section{Summary}

We introduced a DOE with multi-level free-form 3D high-accuracy micro-structure. This DOE was fabricated in $\mathrm{SiO}_{2}$ substrate to withstand a high power and peak laser and have a high transmittance in the visible and near-infrared region. Four beams split by the DOE are focused at a focal length of $1 \mathrm{~m}$. The size of the four spots is $\sim 90 \mu \mathrm{m}$, which is similar to the size of a spot without DOE. The fluctuation of intensity of the four spots is $7.5 \%$. The distance between spots is similar to that of the design of $97 \mu \mathrm{m}$. From these optical results, our multi-level DOE has high performance. Multi-spot processing of a pico-second laser on a copper plate was demonstrated successfully. The processed pattern of twenty-one spots along the optical axis is in good agreement with the optical simulation.

Our multi-level DOE will be useful when using high power and peak power lasers for arbitrary simultaneous multi-spot processing.

\section{Acknowledgments}

The authors would like to thank K. Omata, D. Koshimizu, and Y. Sanbonmatsu of L.P.S. Works Co., Ltd. for assistance with the experiment, and Y. Koyama of Furukawa Denshi Co., Ltd. for assistance with the experiment and simulation. 


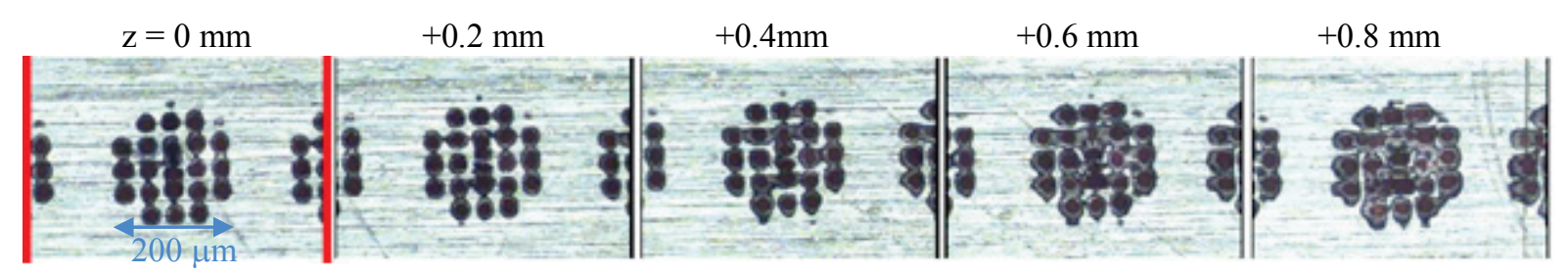

(a) Experimental results of laser processing
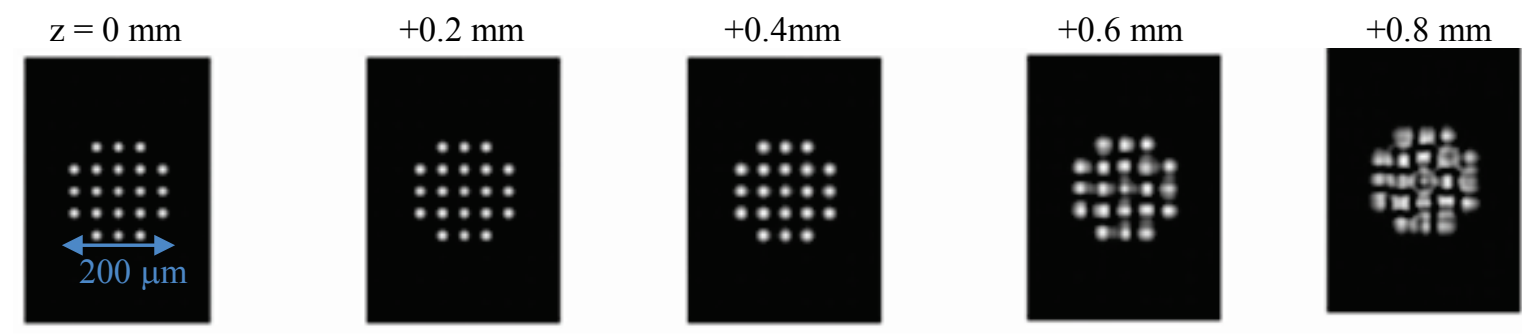

(b) Optical simulation of multi-spot irradiation

Fig. 10 Multi-spot patterns by using the DOE.

\section{References}

[1] Y. Kuroiwa, N. Takeshima, Y. Narita, S. Tanaka and K. Hirao: Opt. Exp., 12, (2004) 1908.

[2] K. Ebata, K. Fuse, K. Kurisu, T. Okada, T. Hirai and H. Nanba: Prod. SPIE, 4400, (2001) 152.

[3] D. C. O'Shea, T. J. Suleski, A. D. Kathman and D. W. Prather: Diffractive Optics: Design, Fabrication, and Test, SPIE Tutorial Texts in Optical Engineering Vol. TT62. (SPIE PRESS, Washington, 2003)

[4] Y. Kuroiwa, N. Takeshima, Y. Narita, S. Tanaka and K. Hirao: Proc. SPIE, (2004) 5539.
[5] N. C. Roberts: Appl. Opt. 31, (1992) 3198.

[6] G. J. Swanson and W. B. Veldkamp: Opt. Eng., 28, (1989) 1908.

[7] A. Hamano, A. Yamada, T. Takada and Y. Usuki: Proceedings of the $2^{\text {nd }}$ Advanced Lasers and Photon Source, (2013) 83.

[8] G. Gal, B. Herman, W. Anderson, D. Shough, D. Prudy and A. Berwick: Pure Appl. Opt. 3, (1994) 97.

(Received: March 13, 2014, Accepted: September 13, 2014) 